\title{
Analysis of Applying UCD in Android App Design
}

\author{
M.Y. Fan \\ Software Engineering Department \\ Beijing Information Technology College \\ Beijing, China
}

\begin{abstract}
Every function of product comes from the demand of user.Designers can use UCD philosophy get more effective information and true requirements from users. This paper first discusses the characteristics of UCD, and why it should be used in design. After comparing Android devices and desktop computers, this paper listed outconsiderations for Android devices in design. Then it gives some suggestions on steps and tools for design Android app in UCD.
\end{abstract}

\section{Keywords-UCD; android; App; design}

\section{INTRODUCTION}

In the software industry, there is a term "user centred design", which is short for UCD and is being thrown about pretty often. If designers could use UCD as its philosophy to design, the result would be accepted easily by users. This paper discusses what is UCD, why to use UCD and how to use UCD in Android app design.

\section{OUTLINE OF UCD}

\section{A. What Is UCD}

According to the voice of wiki, UCD is a process (not restricted to interfaces or technologies) in which the needs, wants, and limitations of end users of a product, service or process are given extensive attention at each stage of the design process. [1]

There are also some other statements about UCD, for example, UCD means making user as the center when designing[2] on baidu. In detail, the design decision will center on user experience, and emphasize the priority of the user.

The main difference between UCD from other design philosophies is that user-centered design tries to optimize the product around users, including how it is used by users, what it is wanted by users, even when it is needed to use by them, instead of forcing the users to change their habit to accommodate the product.

Please keep a second copy of your manuscript in your office. When receiving the paper, we assume that the corresponding authors grant us the copyright to use the paper for the book or journal in question. Should authors use tables or figures from other Publications, they must ask the corresponding publishers to grant them the right to publish this material in their paper.

\section{B. Why Is UCD}

Henry Ford once said the secret to success is to understand the point of view of others. Generally, the final users are not the designers, so whether it is good enough for users is decided by themselves, but not the designers. Just like a person who wears shoes, nobody else knows whether the shoes are comfortable for him, only the man could get the idea of it. So if the designers want to make a perfect result, they must put themselves in the mind-set of the users.

From the point of view of generating of a good product, it must be driven by users' requirements, and here, the users could be a common person, or a department of the government, or another company, or just the invisible market, etc. It's found that in the early of product design, the target requirements could be understood much better, the sooner the better.

Some studies have shown that, if the users are conveyed that the product has paid more close attention to them, they could accept the product more easily. Meanwhile they could stand the defects of the product to a greater extent. Only the product is designed in a user-centred way, the users will have such good experiences.

So, no matter what kind of product is designed, if it wants to get appreciation from users, get good evaluation from users, get profits from users, it should first show the respect and care for its own users.

\section{FEATURES OF ANDROID DEVICES IN DESIGN}

\section{A. Differences between Mobile Devices and Desktop Computers}

Desktop computers are personal computers intended for regular use at a single location desk or table due to its size and power requirements, mainly consist of computer monitor, keyboard, mouse, and a case that houses the main components of the PC, namely the power supply, motherboard, hard drive and optical drive.

Mobile devices are also known as handheld computers or simply handheld, are small, handheld computing devices, typically having a display screen with touch input and/or a miniature keyboard and weighing less than $0.91 \mathrm{~kg}$. They serve a different purpose in users' lives. In this paper, mobile devices mainly refer to the mobile phones, which can make and receive telephone calls over a radio link while moving around a wide geographic area.

Obviously, mobile devices and desktop computers are very different, no matter from the weight, the components they include, or the way they are used, or where they are used. Nowadays, people also find some differences from the point of view of using information: the mobile devices are mostly used to consume information, whereas the desktop computers are used to create information.

So designing products for mobile devices and desktop computers is different too. Designers need to understand the 
limitations and opportunities of both the technology and the use context so as to create a good product.

\section{B. Considerations of Mobile Devices in Design}

\section{1) Environment}

Just like the name suggests, mobile devices are used on the move. So the environment may be very busy, noisy, and users are easily to be distracted at times. In these situations, apps in the mobile devices can't demand the user's full attention. Whether the app and its components are clearly visible, what the app looks like, and how it can be used easily, all of these design result will have important influence to the users.

\section{2) Multitasks}

Mobile devices especially mobile phones, which are smartphones nowadays, are used for a variety of purposes, including keeping in touch with friends and family members, conducting business, gaming, video, audio, having access to a telephone in the event of an emergency, and so on. They are multitasking devices. The result is that when designing apps on them, "good memory" of apps should be concerned.

Everyone knows that mobile devices run on batteries, they have limited power supply, although recently charging pal could make up some deficiencies. So a good designed app should be optimized to conserve power that devices could have enough battery when they are needed. In some special cases, such a good design could decrease serious troubles or even could save a life.

\section{3) Unsteady network}

More often, apps in mobile devices needs to be designed to use the network, at this time, the designers must take "drop network connection" into consideration, and reduce various threatens with it as much as possible.

\section{4) Paying Data}

Data, the important role in today's life, is not free for apps in mobile devices. When app is designed, it should try to limit data transfer only to necessary operations.

Touch screen is the main control interface of most mobile devices, which creates a set of new operating ways for apps. For example, people can control apps with gestures. Just because of gestures, a lot of usability problems should be concerned during design, such as which gesture should be used better, whether the gesture is naturally discoverable enough.

In a word, lots of factors should be considered during design for mobile devices, absolutely they are not limited to the above aspects.

\section{Challenges and Opportunities of the Android Devices in Design}

\section{1) Challenges from Hardware}

The challenges of Android devices in design always start with the hardware, it's just because Android is Open Source, so that nobody can stop anyone else from putting Android on anything they want. Let's take phone for example, Android can run on from low-end smartphones with small displays, barely any memory, and cheap CPUs, to the latest super phones with high-density displays, high-end multi-core GPUs, CPUs, and large memories. Besides, it also runs on tablets, even on TVs, washing machines, glasses and wristwatches. From this point of view, how can designers make one app be suitable for possible devices?

\section{2) Challenges from Android Versions}

The challenges also come from the different versions of Android. It's well known that Google uses different names to indicate different versions of Android OS. And different versions have different API levels. Sometimes because of minor updates, different versions have the same version name, but with different API level. So far, there are 12 names of Android versions, which correspond to 21 API levels.[3]Make the app cover all of the Android versions is a good idea, but it's not always possible. So when the app is designed, which version should it target?

Even with the same version of API level, different devices manufactured by different manufacturers also have different features.

\section{3) Opportunities of Android}

However, just like everything has two sides, the grounding reason that leads to challenges has just provided some opportunities for designers. As above mentioned, Android is Open Source. First, the core part of Android OS is the Linux kernel, which is built by a massive Open Source community and distributed under the GPL, which anyone can take the Linux code and redistribute it, modify it, and even sell it. Second, the framework of Android is built by Google and distributed under the Apache license, which allows distribution of binaries without releasing source code.

What does Open Source mean for designers? They can access to the source code of Android internals, so that when necessary, they can use some basic features to build what they want to implement. Moreover, massive Android communities provide lots of libraries, forums and mature apps, which are much more helpful for design prototyping.

\section{DESIGN FOR ANDROID APP's USER}

\section{A. Steps for Design Android App in UCD}

Since Android devices have so many challenges for design, designers must value the users' demand first. Because different users have different cultural backgrounds, and different habits of using apps, designers should be aware of who the users are, when the users would like to use the app, and even how the users like to use it.

In Figure 1, some key steps are listed out for design Android app in UCD. 


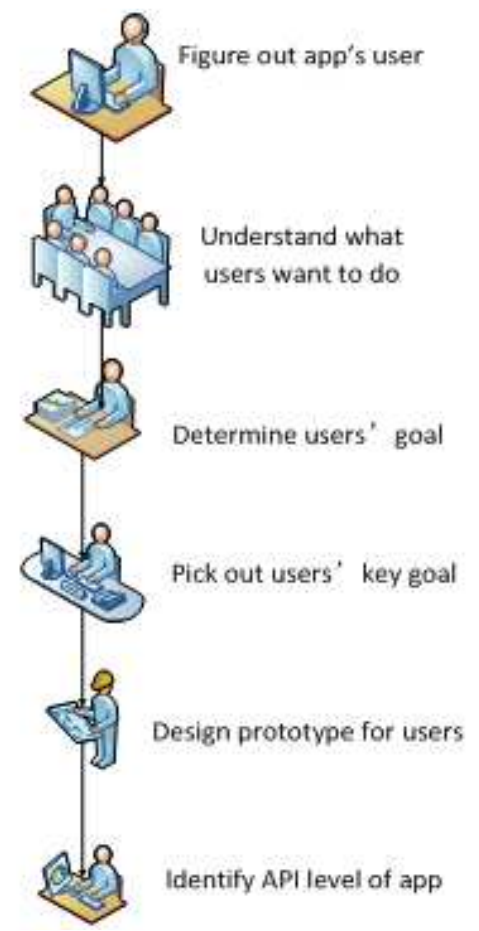

FIGURE I. STEPS FOR DESIGN ANDROID APP

\section{B. Some Tools for Design Android App}

The following discusses some tools that are frequently used in design.

\section{1) Traditional Pencil and Paper}

Pencil and paper are traditional tools for design, which are easy to get and the designed result is easy to be modified. But at some point in the design process it is more convenient to start with some digital tools.

\section{2) Eclipse}

For design, no matter Android app or other software, Adobe's products' names are always mentioned. But for design Android app, there is a special tool named "Eclipse".

Google has chosen Eclipse as the platform for Android tools for a long time. It can not only build UI, but also can be used for design. The obvious benefit of using it is that the design result can be run on real devices, which could be shown to users and get instant feedback from them.

\section{3) Other Digital Tools}

But if designers are not familiar with it, there are also other alternative digital tools, such as AxureRp, BalsamiqMockups, Pencil Project and Prototype Composer[4], etc. All of them could provide different design results with various styles, choose what you want.

\section{SUMMARY}

All above, there are many challenges of Android devices in design, but from the point of view of user, with a right approach, and under the help of some capable tools, it's also possible to get better design result for Android devices.

\section{REFERENCES}

[1] [1] Wikipedia Information on http://en.wikipedia.org/wiki/Usercentered_design

[2] [2] Android Version History http://en.wikipedia.org/wiki/Android_version history

[3] [3] Rapid Prototyping Tools' Comparison on http://www.cr173.com/html/29758_1.html

[4] Information on http://baike.baidu.com/link?url=2e91 pqXzgfQZ8iJsoNmPc5PKGetP9NcbxPsijG4ImIbFmRQGHBPhTYqBHIYxCWCnr Wj3NMkG5OkT96jbKcto_

[5] [4] JuhaniLehtimaki: Smashing Android UI (A John Wiley and Sons, Ltd, Publications, US 2013). 